\title{
Comentarios y notas para una...
}

\section{ге - арагіс í́n}

commentaries and notes on a re appearance

A г mando 0 у аг zún $K$. *

<Resumen>

El autor toma como pretexto el re-lanzamiento de De Arquitectura, para reflexionar en torno a la difícil tarea de publicar los haceres de nuestra disciplina

$<$ Abst ract $>$

The author takes advantage of the I aunching of De Arquitectura new stage to comment on the difficulties of publishing works about our di scipline.

Ciertamente que el mundo editorial es <Palabras clave> REVISTAS DE ARQUITECTURA / PUBLICACIONES PERIÓDICAS DE ARQUITECTURA / ARQUITECTURA CONTEMPORÁNEA

\section{$\langle$ Key words>}

ARCHITECTURE | OURNALS / ARCHI TECTURE PUBLI CATI ONS / CONTEMPORARY ARCHI TECTURE un mundo fascinante y a la vez complejo. Si en países con mayor desarrollo es ARS. dificultoso poder editar, en América Latina y especialmente en Chile, constituye un gesto de resistencia heroica, como lo comentara en alguna ocasión el Arqto. Rafael Moneo, Premio Pritzker, refiriéndose a la desaparecida revista

Es por ello, que la reciente presentación realizada el 18 de enero en la Confitería Torres, de la re-diseñada revista "de Arquitectura, de la Facultad de Arquitectura y Urbanismo de la Universidad de Chile", dirigida por el arquitecto y profesor Constantino Mawromatis, es motivo de gozosa celebración.

Esta revista vio la luz del día en 1990, en el marco de la celebración de los 140 años de la Escuela ${ }^{1}$, bajo el Decanato de don Mario Recordón y la dirección del profesor Alberto Sartori, contaba con un Consejo Editor formado inicialmente por: L. Goldsack, A. Hernández,
H. Montecinos, M. I. Pavez, Oscar Ortega e Isabel Tuca, al cual, se incorporaría en el n5 $H$. Eliash y $E$. Lyon, así como, en el $n^{\circ} 6$ el mismo C. Mawromatis.

Diez años, once números, más cinco años de silencios silentes, esa es la triste realidad del pasado reciente de esta joven publicación.

Apoyamos y aplaudimos esta iniciativa del profesor Mawromatis y este grupo de inquietos colaboradores, de re-tomar, re-fundar y hacer re-nacer esta alicaída publicación, ya que, no es nada común encontrarnos con iniciativas editoriales que sugieran un riesgo tan descomunal, como el que entraña esta "voluntariosa empresa", la cual, se ve agravada, en primer lugar, por las dificultades de financiamiento y en segundo lugar por "la astenia cultural" generalizada que vive un gran segmento de nuestros estudiantes pirquineros ${ }^{2}$, aspectos que en cierta medida determinan el tiraje de esta publicación y en su caso, de manera significativa.

Ex-Coordinador de la Unidad de Extensión, FAU/UCH Director MVADC.

$\mathrm{N}$ de la R de la Escuela de Arquitectura de la FAU. Universidad de Chile.

N. de la R. aquí el autor hace referencia a quienes se juntan en las pircas de la Facultad, en donde se bebe y se fuma de más. 
Estos 500 ejemplares, constituyen el vivo testimonio de esa realidad descrita aquí, haciéndose necesario una red de comunicaciones e intercambios de todas las Escuelas existentes del país, tanto estatales como privadas. ¡Por una vez!, es hora de actuar con visión de futuro y no de manera obtusa, como se ha hecho hasta ahora, en aras a poder relacionarnos y divulgar nuestra "cultura" por lo menos en el territorio nacional, eliminando esta actitud tan cerradamente marcada de centralismo que tenemos los capitalinos y que no conduce evidentemente a nada, sino, "al ostracismo perpetuo".

Pero, de cualquier manera y a pesar de todas las posibles vicisitudes que se hayan vivido en la génesis de ésta y otras tantas y tantas publicaciones y revistas nacionales 0 internacionales de esta naturaleza, siempre detrás de ellas ha habido un arquitecto o un grupo de ellos, desde, Vitruvio, Palladio y Vignola a Le Corbusier.

Los arquitectos del siglo xx no solamente han sido constructores, sino, también ideólogos que han explorado tanto los problemas implícitos en la misma arquitectura como los que radican en sus bases sociales humanas y económicas. Así se explica la cantidad e importancia de los programas, manifiestos y publicaciones que han sido firmados y editados por estos dentro del Movimiento de la Arquitectura Moderna para definir su posición frente a los mismos.

El fin de siécle tiene en Henry van de Velde (Amberes 1863) un teórico y un portavoz experimentado en el arte del discurso y la controversia. Sus primeras publicaciones de orientación aparecieron a mediados de 1890 a partir de 1896 su nombre esta fuertemente ligado al concepto de "L'Art Nouveau". En el curso de un viaje a Alemania en el invierno de 1900-1901, proclama, en Kunstgewerblichen Laienpredigten su estética funcional, la estética de la "forma pura".

En 1917 el Grupo Neoplásticista De Stijl liderado por Theo van Doesburg publica la revista del mismo nombre, con la colaboración de otros artistas radicales, tales como,
Mondrian, van der Leck, Huszar, Wils, Kok, Hoff, Vantongerlo y van Esteren. Un año después, en 1918, aparece el primero de los cinco Manifiestos del grupo, su principal objetivo: la combinación orgánica de arquitectura, plástica y pintura en una formación clara, elemental, sin sentimientos.

El Architektur-Programm de Bruno Taut fue publicado como "un folletín" en Navidades del año 1918, con la aprobación del Arbeitstrat für Kunst.

En abril de 1919 el Arbeitstrat für Kunst presenta en Berlín una Exposición para Arquitectos Desconocidos, además editan "un folleto" de cuatro páginas, el cual presenta tres textos con carácter de Manifiesto firmados por Walter Gropius, Bruno Taut y Adolf Behne. En el momento de la exposición, Gropius ya había sido nombrado director de las Vereinigten ehemals Grossherzoglichen Schule für Bildende Kunst und Kuntsgewerbe (Escuelas Unidas de Bellas Artes y Artesanía) de Weimar, a la cual se le redefine su nombre, pasando a llamarse Staatliches Bauhaus. Este texto en lo esencial equivale al primer Manifiesto de la Bauhaus.

En Francia Auguste Perret funda la revista "Tecniques \& Architecture"; siendo miembros fundadores Le Corbusier y Le Ricolais. Muy posteriormente la diseñadora de esta publicación es Michelle Huidobro, hija de Émile Duhart (recientemente fallecido) casada con Borja Garcia Huidobro, hoy, Borja Huidobro.

Entre los años 60 y 70 el gran referente en la cultura arquitectónica de América Latina lo constituye la revista francesa l'architedure d'aujourd'hui, fundada por el arquitecto André Bloc.

El año 1957 Yona Friedman arquitecto húngaro, se establece en París donde funda el Grupo de Estudios de la Arquitectura Móvil, generando las bases de su teoría, la cual, impulsará a ARCHIGRAM, grupo de jóvenes ingleses insatisfechos, en el Invierno de 1961 a iniciar una de las aventuras más espectaculares que ha vivido la "arquitectura inglesa", ellos editaron una publicación titulada
Archigram, el primer número fue simplemente "una hoja" y contenía unos poemas de David Greene, se vendieron solamente 300 ejemplares, todo el mundo creyó que Archigram moriría de muerte natural. El segundo número salió un año (y 3 concursos) después; tras esta extraordinaria publicación estaban también Ron Herron, Dennis Crompton, Michael Web, Warren Chalk y el incombustible Peter Cook, quién diera clases en la Arquitectural Association (fundada en 1847, siendo la más antigua de las Escuelas de Arquitectura de Gran Bretaña) sobre todo en el período de oro de ésta, aquel que dirigió Alvin Boyarsky. En la actualidad Peter Cook es director de la Bartlett School of Architecture.

talia no es ajena a estos temas; la revista Casabella fue fundada por Guiseppe Pagano, y en el período del 53 al 64, Ernesto Rogers N. dirigió a un grupo de redactores: V. Gregotti, A. Rossi, G. Aulenti, C. Aymonino, G. de Carlo, todos ellos destacadísimos arquitectos de los 75 al 90. Rogers es socio del grupo BBPR, autores de la polémica Torre Velasca de Milán en 1954-1958 (tío de R. Rogers). La revista también fue dirigida por el argentino Tomás Maldonado, quién fuese Director de la Escuela de Diseño de Ülm, en Alemania. Posteriormente también lo ha sido V. Gregotti, autor del proyecto para la Universidad de la Calabria, en Sicilia, un proyecto lineal, que tiene aproximadamente $3,5 \mathrm{~km}$ de longitud.

En 1927 Gio Ponti funda la revista Domus, siendo coautor junto a Pier Luigi Nervi de la Torre Pirelli, en Milán el año 1958. En el último tiempo Alessandro Mendini ha sido su director, con quién a tenido un cambio de imagen, sobre todo en la portada.

Otras significativas revistas italianas de referencia son: Lotus, fundada en 1963 y que cuenta con un Comité Editor en donde estaban Gae Aulenti, Oriol Bohigas, Mario Botta, Kenneth Frampton, Vittorio Gregotti, Joseph Rykwert, Christian Norberg-Schulz. También esta Parámetro, que en su Comité Directivo contaba con Giancarlo de Carlo y Ludovico Quaroni. Otra publicación que ha trascendido y ha sido notable referente, especialmente en España, es la revista Controspazio, fundada 
en junio del 1969 y dirigida por el exuberante arquitecto y crítico Paolo Portoghesi, habiendo tenido en la redacción a otros tantos destacados arquitectos italianos, como: Franco Purini, Alessandro Anselmi, Francesco Cellini, Francesco D'Amato, Ezio Bonfanti y Laura Thermes. El primer número estuvo dedicado a dos proyectos de Louis Kahn, Dhaka y Venecia Ch. Norberg-Schulz presenta un ensayo sobre el "concepto de lugar"; Ezio Bonfanti escribe sobre "la Autonomía de la Arquitectura"; Paolo Marconi analiza el Octavo libro inédito de Sebastiano Serlio, quién difundiera en Francia el Renacimiento Italiano y que trata sobre Arquitectura Militar, y Virgilio Vercelloni escribe sobre la última obra de Mario Ridolfi. Que notables y descriptivos dibujos realizaba Ridolft a quién Enric Miralles le profesaba profunda admiración. Si nos detenemos a observar dibujos de ambos podemos concluir en la fuerte influencia de éste sobre Enric.

Hasta la década de los 80 la Editorial Gustavo Gili, de Barcelona-España ejerce un amplio monopolio e influencia editorial en América Latina, habiéndose preocupado demasiado poco o nada de la Arquitectura Latinoamericana. Hasta el año 2000, publicó un solo libro sobre este tema escrito por Francisco Bullrich, e impreso en Argentina con fecha del año 1969, siendo una edición exclusiva para Europa. El año 94 edita en la colección Catálogos de Arquitectura Contemporánea la obra del arquitecto brasilero Paulo Mendez da Rocha, tres años después la colección Portafolio se inaugura con la publicación de Mathías Klotz, aunque se cuenta que estaba previsto hacerlo con la obra de Shigeru Ban. Klotz se constituye así, en el primer arquitecto chileno a quién una editorial le publica su obra de manera exclusiva.

Pero, han trascurridos largos años y esta situación de monopolio que ha ejercido la GG, se perdió. Hoy existen otras editoriales tan potentes como esta en España, tenemos a Actar, Blume, Arco Editorial, Tusquet Editor y Ediciones Destino en Barcelona, en Madrid esta Xarait Ediciones.

España ha vivido toda suerte de aventuras editoriales, desde la revista Nueva Forma, dirigida por Juan Daniel Fullaondo, que era financiada por la empresa Huarte, dando término en el número 111 a su singladura. Nueva Forma se constituyo entre los 60 y 80 en una revista de alto nivel intelectual, incorporando filosofía, poesía, escultura y pintura, dio a conocer a James Stirling, Claude Parant, Paul Virilio, a los hermanos León y Robert Krier a Rafael Canogar, Eduardo Chillida y otros. Estuvo permanentemente atenta a lo que sucedía en el territorio español. Fue una revista osada en una época de plena dictadura franquista (Franco falleció un 20 de noviembre de 1975). En Barcelona se editó la revista Arquitecturas Bis, en mayo de 1974 apareció su primer número, bajo la dirección de Rosa Regás contaba con un Consejo de Redacción donde estaban los más prominentes arquitectos catalanes: Oriol Bohigas, Federico Correa, Lluís Doménech, Helio Piñon, Manuel de Solá Morales, Oscar Tusquets, más el vasco Luís Peña Ganchegui y el navarro Rafael Moneo, el filósofo Tomás Llorens y el diseñador gráfico catalán Enric Satué, quien fuese posteriormente Premio Nacional Español de Diseño. Esta revista desde sus inicios fue una revista diferente, con un prestigioso Consejo, fue cobijo de importantes ensayos reflexiones, críticas y comentarios de arquitectura y su número 52 fue anunciado como el número final extraordinario, esto es en pleno invierno de diciembre de 1985. Comenzó costando 50 pesetas y término en 1.500, habían pasado casi 12 años y tras sí dejaba una estela de revistas hermanas que perpetuaban su imagen: Skyline, Express y Architectures de Nueva York, Arq de Santiago de Chile (en su primera etapa), Arquetype de San Francisco, Section A de Montreal, In Architettura de Palermo. Desde Barcelona se editan la revista Quaderns, órgano oficial del Colegio de Arquitectos de Cataluña, que tuvo su etapa importante con la dirección de Josep Lluís Mateo, y la revista ON, dirigida por $\mathrm{M}^{\mathrm{a}}$ Carmen Ferrer.

En Madrid, Arquitectura, una publicación periódica del Colegio Oficial de Arquitectos de Madrid fundada en 1918, tuvo su momento álgido bajo la dirección de los destacadísimos arquitectos y socios en la vida privada
Estanislao Pérez Pita y el fallecido Jerónimo Junquera. En la Actualidad El Croquis dirigida por Richard Levene y Fernando Márquez, es sin duda, una de las editoriales importantes, inclusive, cuenta con una Galería de Exposiciones, de igual manera que la revista japonesa GA que dirige en Tokio el conocido fotógrafo Yukio Futagawa. También desde Madrid se editan A\&V y Arquitectura Viva, ambas dirigidas por Luís Fernández Galiano, y Diseño Interior

En el país Vasco, el Departamento de Urbanismo, Vivienda y Ambiente de la Dirección de Arquitectura del Gobierno Vasco, edita una publicación bajo el título Hirigintza eta Eraikintzari Buruzko AldikariaTecnología y Arquitectura, esta publicación es bilingüe, castellano y euskera. Después de varios años de dirigirla Javier Mozas y Aurora Fernández, optaron por editar una publicación independiente y sacaron la revista a+t, arquitectura y tecnología, que se edita desde Vitoria-Gasteiz.

La Delegación de Almería del Colegio Oficial de Arquitectos de Andalucía Oriental edita una publicación bajo el título Documentos de Arquitectura desde 1987.

Tan importante ha sido la aparición de revistas en España, así como, su desaparición: Jano Arquitectura, Ardi, De Diseño, Carrer de la Ciutat, 2C Construcción, A30, Hogar y Arquitectura, Diversa, Común Arquitectura y Tecnología, Hogares Modernos y otras tantas que constituyen una larga lista de fenecidas publicaciones, pero, la que pudo ser la más internacional de éstas la revista Jano, con un Consejo Editor donde estaban James Stirling, Oriol Bohigas, Oscar Tusquets, Mario Botta, Clorindo Testa y alguno más que no recuerdo, lanzó un número 0 de presentación y después hubo solo silencio.

En la Argentina, a inicios de los 60 surge la revista Summa dirigida por Lala Méndez Mosquera, para luego editar los famosos cuadernos Summa-nueva visión, que dirigiera la arquitecta y crítica Marina Waisman. Esta última publicación tuvo varias y notables series: 


\section{- El diseño del entorno humano}

- Enfoques crítico-históricos

- Tendencias de la Arquitectura actual

Posteriormente la editora Lala Méndez Mosquera edito unos cuadernillos titulados colección sumarios.

Para el mundo cultural nacional estas publicaciones fueron de suma importancia, ya que, a Chile la información en esos años llegaba con gran retraso, pasaban varios años antes de estar al día en las novedades que se sucedían más allá de nuestras fronteras. En ciertos momentos estas publicaciones se constituyeron en la única fuente de información, aquí y en América del Sur.

La Sociedad Central de Arquitectos Argentinos fue fundada el 18 de marzo de 1886 y un 15 de abril de 1904 fundaban la publicación SCA Revista de Arquitectura, vigente en la actualidad.

En Brasil la Editora Pini Ltda. edita desde 1948 la revista Arquitetura \& Urbanismo, ésta es una empresa familiar, en donde la Editora general es la conocida Haifa Y. Sabbag. Otra revista es Modulo que dirige Oscar Niemayer, ésta es una publicación autorreferente.

En Perú, el arquitecto Fernando Belaúnde Terry funda en 1937 la revista El arquitecto Peruano, más tarde sería Presidente del Perú en dos oportunidades. Hace no muchos años que Frederick Cooper, socio de Graña y Nícolini, importantes profesionales peruanos, dirige la publicación Arkinka.

En Chile hemos tenido pocas publicaciones, la Sociedad Cooperativa A.U.C.A., en diciembre de 1965 editó una revista independiente bajo el nombre de AUCA, revista sobre Arquitectura, Urbanismo, Construcción y Arte, que agrupo a importantes arquitectos de la Universidad de Chile, entre los accionistas se contaban: Juan Martínez, Sergio González, Mario Recordón, Jaime Bendersky, Ana Mª Barrenechea, Raquel
Eskenazi, Raúl Farrú, Abraham Schapira, Miguel Lawner, Juan Cárdenas, José Covacevic, Osvaldo Cáceres, Alberto Sartori, Julio Mardones, Pedro Iribarne, Francisco Ehijo, Ricardo y Juan Tapia Chuaqui, León Messina... y otros tantos más. Al término de los 80, habían editado 51 números. Su desaparición se hizo notar en el medio, sobre todo entre los estudiantes de la época; era nuestro gran referente. Revista AUCA supo generarse un espacio de comunicación muy importante.

El Colegio de Arquitectos edita la revista $\mathrm{CA}$, que desde el $\mathrm{n}^{\circ} 16$ fue dirigida por Jaime Márquez hasta mediados del 2002. Hoy, tiene otra orientación y dirección.

En agosto del año 1977 el grupo C.E.D.L.A. edita una singular publicación que luego daría vida a la revista ARS del mismo Centro y que aparecería en julio de 1978. Después de 10 números, dejó de existir, cuando tenía vocación de ser una Revista Latinoamericana de Arquitectura, era mayo de 1988.

Pero, sin lugar a dudas la editorial más importante de América Latina es Ediciones $A R Q$, de Escuela de Arquitectura de la Pontificia Universidad Católica, que edita la revista $A R Q$ fundada en 1980 , bajo la dirección de la importante activista cultural ex Decana de dicha Escuela, la arquitecta Montserrat Palmer. La editorial tiene y mantiene una intensa actividad que realmente es ejemplar en Chile y América Latina.

Durante casi cuatro años el arquitecto argentino Tomás Dagnino edito TodoobrasChile periódico gratuito, financiado por las empresas que incorporaban su publicidad, además se repartía en varias Escuelas de Arquitectura del país, se publicaron 41 números, que dieron cuenta de la realidad de momento, con entrevistas regulares a algunos arquitectos del medio profesional en activo. Su desaparición se extraña profundamente en el medio. Hoy, el arquitecto Dagnino dirige el Centro TOD, en Buenos Aires y edita Todoobras-Argentina.
Otras dos interesantes publicaciones son: 180 - Cientochenta, de la Facultad de Arquitectura, Arte y Diseño de la Universidad Diego Portales, de la cual es decano Mathías Klotz. La revista tiene un carácter universitario y es editada por Hernán Garfias, antiguo editor de la desaparecida Diseño, que tanto le debe a la revista De Diseño editada en Barcelona, dirigida por Juli Capella y Quim Larrea.

La otra publicación a la cual deseo referirme, es la poco conocida aún ARTEOFICIO-cuadernos de la Escuela de Arquitectura de la Universidad de Santiago, esta revista si bien, de modesta factura tiene una gran fuerza discursiva ya que los escritos de algunos de los colaboradores, constituyen notables ensayos. El valor propositivo de la revista, permite dar espacio a nuevas voces, tan necesarias en un medio tan complejo como este.

- Y cuando teníamos la mitad de un verano tórrido, el Museo de Arte Contemporáneo, en su nueva sede de Matucana, el 20 de enero presentaba la más joven de las revistas en Chile, SPAM-arq., una publicación independiente, patrocinada por el MAC y la Escuela de Arquitectura de la FAU de la UCH. De una gráfica pregnante, su apuesta es constituirse en una plataforma para la observación, investigación y difusión de las nuevas condiciones que configuran la ciudad actual, a partir de un enfoque transdiciplinar, como lo enuncian en su declaración de principios.

No cabe dudas, de que este recorrido que se inició sin saber que destino final iba a tener, me ha permitido unir "el allí, con el aquí", y viajar en el tiempo, en ese tiempo que inexorablemente pasa y que no perdona. 